UCRHEP-T579

June 2017

\title{
Anomalous Leptonic U(1) Symmetry: Syndetic Origin of the QCD Axion, Weak-Scale Dark Matter, and Radiative Neutrino Mass
}

\author{
Ernest $\mathrm{Ma}^{1}$, Diego Restrepo ${ }^{2}$, and Óscar Zapata ${ }^{2}$ \\ 1 Department of Physics and Astronomy, \\ University of California, Riverside, California 92521, USA \\ 2 Instituto de Física, Universidad de Antioquia, \\ Calle 70 No. 52-21, Apartado Aéreo 1226, Medellín, Colombia
}

\begin{abstract}
The well-known leptonic U(1) symmetry of the standard model of quarks and leptons is extended to include a number of new fermions and scalars. The resulting theory has an invisible QCD axion (thereby solving the strong CP problem), a candidate for weak-scale dark matter, as well as radiative neutrino masses. A possible key connection is a color-triplet scalar, which may be produced and detected at the Large Hadron Collider.
\end{abstract}


Introduction : In the standard model (SM) of quarks and leptons, there are four automatic global symmetries: baryon number $B=1 / 3$ for each quark, lepton number $L_{e}=1$ for the electron and its neutrino $\nu_{e}, L_{\mu}=1$ for $\mu$ and $\nu_{\mu}$, and $L_{\tau}=1$ for $\tau$ and $\nu_{\tau}$. As such, all neutrinos are massless. Given that we now know that neutrinos are massive and mix among themselves, the SM must be extended and $L_{e, \mu, \tau}$ must be replaced with $L=L_{e}+L_{\mu}+L_{\tau}$. Hence $L$ is still a valid global $U(1)$ symmetry if neutrinos are strictly Dirac fermions, but often than not, they are assumed to be Majorana fermions so that $L$ is broken to $(-1)^{L}$, i.e. lepton parity.

Theoretical mechanisms for obtaining Majorana neutrino masses are many [1], but there is no experimental evidence for any one of them. Then there is the dark matter (DM) of the Universe. The SM has no explanation for it, but the intriguing idea exists that it may be connected to the neutrino's mass generating mechanism. In 2006, a simple one-loop radiative mechanism was proposed [2] with dark matter in the loop, called "scotogenic" from the Greek "scotos" meaning darkness. In 2015, it was shown [3] that the dark parity of this model, as well as many others, is derivable from lepton parity. This demonstrates how the leptonic $U(1)_{L}$ symmetry may be extended to include particles beyond those of the SM.

In 2013, it was shown [4] that the well-known spontaneously broken anomalous PecceiQuinn $U(1)_{P Q}$ symmetry [5], which solves the strong CP problem [6] and creates the QCD axion [7, 8], has a residual $Z_{2}$ symmetry which may in fact be dark parity. In this paper, we combine all these ideas to show that, with the proper choice of fermions and scalars beyond the SM, we can have $U(1)_{P Q}=U(1)_{L}$ with the residual dark parity [3] given by $(-1)^{3 B+L+2 j}$, i.e. the well-known $R$ parity of supersymmetry, but not in a supersymmetric context.

To implement this important new insight, i.e. $U(1)_{P Q}=U(1)_{L}$, in a specific model, we choose the singlet-doublet-fermion dark-matter scenario with three additional scalars to obtain scotogenic neutrino masses [9, 10]. This framework is however also adaptable for 
radiative quark and lepton masses [11, 12].

Particles Beyond the SM : The new particles of our model are assigned under $U(1)_{L}$ as shown in Table 1 . Note that the only new fermion which transforms under $U(1)_{L}$ is $D$. As

Table 1: Particle assignments under $P Q=L$.

\begin{tabular}{|c|c|c|c|c|c|c|}
\hline Particle & $S U(3)_{C}$ & $S U(2)_{L}$ & $U(1)_{Y}$ & $P Q=L$ & $B$ & $R$ \\
\hline$D_{L}$ & 3 & 1 & $-1 / 3$ & 1 & $1 / 3$ & - \\
$D_{R}$ & 3 & 1 & $-1 / 3$ & -1 & $1 / 3$ & - \\
\hline$N_{L}$ & 1 & 1 & 0 & 0 & 0 & - \\
$\left(E^{0}, E^{-}\right)_{L, R}$ & 1 & 2 & $-1 / 2$ & 0 & 0 & - \\
\hline$\zeta$ & 3 & 1 & $2 / 3$ & 0 & $1 / 3$ & - \\
\hline$\chi_{1,2,3}$ & 1 & 1 & 0 & 1 & 0 & - \\
\hline$\sigma$ & 1 & 1 & 0 & 2 & 0 & + \\
\hline
\end{tabular}

this anomalous $U(1)_{L}$ is broken by $\langle\sigma\rangle$, the QCD axion appears, together with the residual symmetry $R=(-1)^{3 B+L+2 j}$, which is even for SM particles as well as $\sigma$, but odd for all the other new particles. The axion is thus of the KSVZ type [13, 14] and the domain wall number is 1 , so it is cosmologically safe [15].

The axion decay constant $F_{A}$, i.e. $\langle\sigma\rangle$, is known to be large [16]: $F_{A}>4 \times 10^{8} \mathrm{GeV}$. Hence the singlet $D$ quark is expected to be heavy, unless the Yukawa coupling for $\sigma \bar{D}_{L} D_{R}$ is very small. If this is indeed the case, then $D$ may be produced in pairs at the Large Hadron Collider (LHC), and the $\bar{D}_{L} d_{R} \chi$ term [4] would allow it to be discovered. Alternatively, if a dark scalar doublet $\left(\eta^{+} \eta^{0}\right)$ exists as in the original scotogenic model [2], then the $\bar{D}_{R}\left(u_{L} \eta^{-}+\right.$ $d_{L} \bar{\eta}^{0}$ ) term works as well [17]. On the other hand, if $D$ is very heavy (of order $F_{A}$ ) as expected, then it is impossible for it to be produced at the LHC. In this study, we will consider instead the dark scalar quark $\zeta$ with charge $2 / 3$. Its mass is not constrained and may well be within the reach of the LHC and be produced copiously in pairs through its gluon interaction. 
Two-Component Dark Matter: As shown in Ref. [4], the coexistence of the QCD axion with a stable weak-scale particle allows for a much more flexible two-component theory of dark matter. It relaxes the severe constraints imposed on either component if considered alone. It allows for a solution of the strong CP problem, without having the QCD axion as observable dark matter. Regarding the weak-scale DM particle, it is the lightest particle charged under the residual dark parity $(-1)^{3 B+L+2 j}$, and can be either a real scalar [21, 22, 23] or an admixture of a singlet-doublet fermion [24, 25, 26, 27].

In the scalar case, since $\chi_{1,2,3}$ carry lepton number, they are complex with invariant $\left(m_{\chi}^{2}\right)_{i j} \chi_{i}^{*} \chi_{j}$ terms. However, a $6 \times 6$ mass-squared matrix is obtained because there are also the allowed $\sigma^{*} \chi_{i} \chi_{j}$ terms. There are thus six real scalar eigenstates. Since the mass splittings of the real and imaginary parts of the complex $\chi$ scalars are proportional to $\langle\sigma\rangle$, they are presumably large. Hence fine tuning is required [4] to make one component light and the other heavy, if we want the lightest $\chi\left(\right.$ call it $\left.\chi_{0}\right)$ to be dark matter.

In the fermion case, there are invariant mass terms $m_{E} \bar{E}_{R} E_{L}$ and $m_{N} N_{L} N_{L}$, as well as the allowed mixing terms between $N$ and $E^{0}$ which are proportional to $\left\langle\phi^{0}\right\rangle$. The $3 \times 3$ mass matrix spanning $\left(N_{L}, E_{L}^{0}, \bar{E}_{R}^{0}\right)$ is then of the form

$$
\mathcal{M}_{N E}=\left(\begin{array}{ccc}
m_{N} & m_{L} & m_{R} \\
m_{L} & 0 & m_{E} \\
m_{R} & m_{E} & 0
\end{array}\right)
$$

resulting in 3 Majorana fermion eigenstates, the lightest (call it $N_{0}$ ) is dark matter. Assume for example $m_{L}=m_{R}=m_{V} / \sqrt{2}>0$ and $m_{N}=m_{E}>0$, then the three mass eigenvalues (in increasing magnitude) are

$$
m_{E}-m_{V}, \quad-m_{E}, \quad m_{E}+m_{V}
$$


corresponding to the three mass eigenstates

$$
\begin{aligned}
& N_{0}=N_{L} / \sqrt{2}-\left(E_{L}^{0}+\bar{E}_{R}^{0}\right) / 2, \\
& N_{1}=\left(E_{L}^{0}-\bar{E}_{R}^{0}\right) / \sqrt{2}, \\
& N_{2}=N_{L} / \sqrt{2}+\left(E_{L}^{0}+\bar{E}_{R}^{0}\right) / 2 .
\end{aligned}
$$

In either case, it may only account for part of dark matter, the rest coming from axions. In direct-search experiments, the exchange of $Z$ is irrelevant because $\chi_{0}$ is a singlet, and $N_{0}$ is Majorana. However, the exchange of $h$ (the SM Higgs boson) will contribute. As for relic abundance, beyond those interactions of the minimal models mentioned earlier, we have also the Yukawa terms $\bar{\nu}_{i} N_{0}$, which may also contribute. There are many free parameters in our model to make this work, but it is not our goal to examine them in any detail. After all, these issues have been dealt with thoroughly in those previous studies. Instead, we will focus on the feasibility of finding the scalar quark $\zeta$ which connects the high scale $\left(10^{9}\right.$ to $\left.10^{11} \mathrm{GeV}\right)$ of the axion to the much lower scale $(100 \mathrm{GeV})$ of the dark-matter candidates $\chi_{0}$ and $N_{0}$.

Scotogenic Neutrino Mass: Using the Yukawa terms $\chi\left(\bar{\nu}_{L} E_{R}^{0}+\bar{e}_{L} E_{R}^{-}\right), \bar{N}_{L}\left(\phi^{0} E_{R}^{0}-\phi^{+} E_{R}^{-}\right)$, $N_{L}\left(\phi^{0} E_{L}^{0}-\phi^{+} E_{L}^{-}\right)$, and $\sigma_{2}^{*} \chi_{i} \chi_{j}$, the one-loop diagram of Fig. 1 is obtained, thereby generating

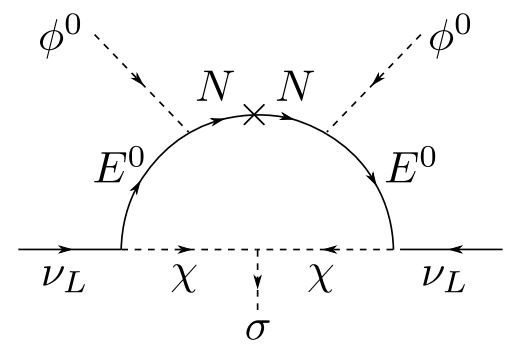

Figure 1: One-loop generation of neutrino mass with $U(1)_{L}$.

three radiative neutrino masses through the spontaneous breaking of $U(1)_{L}[9$, 10]. This idea was previously applied directly to the canonical seesaw mechanism with singlet right-handed 
neutrinos [18, 19], thus equating the axion scale to that of the neutrino seesaw. Here the axion scale enters through $\langle\sigma\rangle$. Our model differs conceptually from the previous use of Fig. 1 because we equate $U(1)_{L}$ with $U(1)_{P Q}$ and let it be spontaneously broken. However, the resulting neutrino mass matrix has the same structure as previous studies and the details are available in those references [9, 10].

In this scotogenic model, the family index is carried by $\chi$, so a possible family symmetry may be considered. Let $\nu_{1,2,3}$ and $\chi_{1,2,3}$ transform as $\underline{3}$ under the non-Abelian discrete symmetry $T_{7}$ for example [20]. The group multiplcation rule of $T_{7}$ is

$$
\underline{3} \times \underline{3}=\underline{3}+\underline{3}^{*}+\underline{3}^{*}
$$

and since $\sigma \sim \underline{1}$ under $T_{7}$, it does not close the loop of Fig. 1. We now need to add three extra scalars $\rho_{1,2,3} \sim \underline{3}$ or $\underline{3}^{*}$ to couple to $\chi_{i} \chi_{j}$ to complete the loop of Fig. 1 . It is then possible for $\left\langle\rho_{1,2,3}\right\rangle$ to be much smaller than $\langle\sigma\rangle$, in which case the lightest $\chi$ may be naturally of the electroweak scale as a dark-matter candidate.

Possible Hadronic Connection : Whereas the heavy color-triplet fermion $D$ connects with the SM through the Yukawa term $\bar{D}_{L} d_{R} \chi$, another possible way is through the color-triplet scalar $\zeta$, with the important terms $f_{D} \zeta \bar{D}_{L} e_{R}+H . c$. as well as

$$
\begin{gathered}
f_{N} \zeta \bar{u}_{R} N_{L}+f_{E} \zeta^{*}\left(\bar{E}_{R}^{0} u_{L}+\bar{E}_{R}^{+} d_{L}\right)+H . c .=f_{N} \zeta \bar{u}_{R}\left(N_{0}+N_{2}\right) / \sqrt{2} \\
+f_{E} \zeta^{*}\left(-N_{0} / 2-N_{1} / \sqrt{2}+N_{2} / 2\right) u_{L}+f_{E} \zeta \bar{d}_{L} E_{R}^{-}+H . c .
\end{gathered}
$$

We assume that $D$ is very heavy, so it decays away quickly in the early Universe to either $e \zeta$ or $d \chi$. Subsequently, either $\chi_{0}$ or $N_{0}$ becomes a component of the dark matter of the Universe, together with the axion.

To test our hypothesis, we propose a search for $\zeta$ at the LHC. It is easily produced, because it is a scalar quark. We assume first that the Majorana fermion $N_{0}$ is dark matter. 
If $f_{N}$ is dominant, then $\zeta$ decays equally to $N_{0}$ and $N_{2}$, with a quark jet in each case. Whereas $N_{0}$ is stable and invisible, $N_{2}$ will decay, i.e.

$$
N_{2} \rightarrow \nu_{i} \chi_{j}+E^{ \pm} W^{\mp}
$$

with the subsequent decay or conversion (if $\chi_{j}$ is virtual)

$$
\chi_{j} \rightarrow \nu_{k} N_{0}+\ell_{k}^{ \pm} E^{\mp}
$$

and

$$
E^{ \pm} \rightarrow W^{ \pm} N_{0}
$$

Most events are then of the type 2 jets + missing energy. They are thus analogous to scalar quark pair production with decays to a quark and a neutralino in supersymmetry. We can thus borrow from the existing studies of supersymmetric scenarios to put a bound on $m_{\zeta}$ as a function of $m_{N_{0}}$ and $m_{N_{2}}$.

If $f_{E}$ is dominant, then $\zeta$ decays equally to $N_{0,1,2}$ and $E^{+}$, with a quark jet in each case, as shown in Eq. (7). Whereas most $N_{0,1,2}$ decays are invisible, $E^{+}$decays according to Eq. (10). This is analogous to a squark decaying to a quark and a chargino which then decays to a $W$ and a neutralino in supersymmetry. If we focus on the leptonic decay of $W$, then the final states of $\zeta \zeta^{*}$ production at the LHC may also include 2 jets $+\ell^{ \pm}+$missing energy and 2 jets $+\ell_{1}^{+} \ell_{2}^{-}+$missing energy.

Consider the alternative case that the real scalar $\chi_{0}$ is dark matter. This means that the fermions $N_{i}$ are heavier. The decay of $\zeta$ to $u+N_{i}$ will have another step, i.e.

$$
N_{i} \rightarrow \nu_{j} \chi_{0}
$$

which are invisible. The signature is again 2 jets + missing energy, but now there are two relevant masses, $m_{N_{i}}$ and $m_{\chi_{0}}$. If $m_{N_{i}}$ is close to $m_{\zeta}$, the kinematics will be quite different from the supersymmetric analog discussed previously where $N_{0}$ is dark matter. The quark 
jets will be soft and could miss the cut on their momenta. In that case, if $f_{N}$ dominates in Eq. (7), the signal is just missing energy.

If $f_{E}$ dominates, we have again the decay of $\zeta$ equally to $N_{i}$ and $E^{+}$. For the latter, the second step is now

$$
E^{+} \rightarrow \chi_{0} \ell_{i}^{+}
$$

The final states of $\zeta \zeta^{*}$ production at the $\mathrm{LHC}$ will again include 2 jets $+\ell^{ \pm}+$missing energy and 2 jets $+\ell_{1}^{+} \ell_{2}^{-}+$missing energy. However, since the charged leptons come directly from $E$ decay, their numbers are not diminished by the branching fraction of $W$ to leptons as in the case where $N_{0}$ is dark matter. Also, if $m_{E}$ is close to $m_{\zeta}$, the jets may be too soft to be observable. In that case, we will only find leptons + missing energy.

$\underline{L H C \text { Signatures }}$ : We will discuss first the case of fermion dark matter, where we have the main collider signature of two jets plus missing transverse energy: $2 j+E_{\mathrm{T}}^{\text {miss }}$. The further decay of $E^{+}$to $E^{0}$ via a $W$-boson only increases the number of soft objects so that the main signal is still just $2 j+E_{\mathrm{T}}^{\text {miss }}$. This contribution, through the corresponding quasi-degenerate $E^{+}-E^{0}$ states, are usually already included in the analyses to be discussed below.

Regarding the signal $2 j+E_{\mathrm{T}}^{\mathrm{miss}}$, it has been already studied by ATLAS and CMS in the context of simplified supersymmetric scenarios searching for squarks decaying into a quark and neutralino. In the case of fermion dark matter, our branching fraction is approximately 100\%. The results for the production of a single squark reported by CMS [28] based on 35.9 $\mathrm{fb}^{-1}$ at $13 \mathrm{TeV}$ are thus fully applicable in our case and reproduced in Fig. 2 (solid line). They allow us to exclude, for example, $m_{\zeta}$ up to $1.0(0.8) \mathrm{TeV}$ for $m_{N_{0}}=100(400) \mathrm{GeV} 1$ The exclusion limit at $95 \%$ confidence level on the cross section of direct production of $\zeta$ pairs (color bar) from [28] is shown in Fig. 2, where the region below the solid line corresponds to

\footnotetext{
${ }^{1}$ It is worth noticing that contrary to the standard scenario of singlet-doublet fermion dark matter we can have now doublet-like fermion dark matter component less that $1 \mathrm{TeV}$ while still being compatible with direct detection constraints.
} 


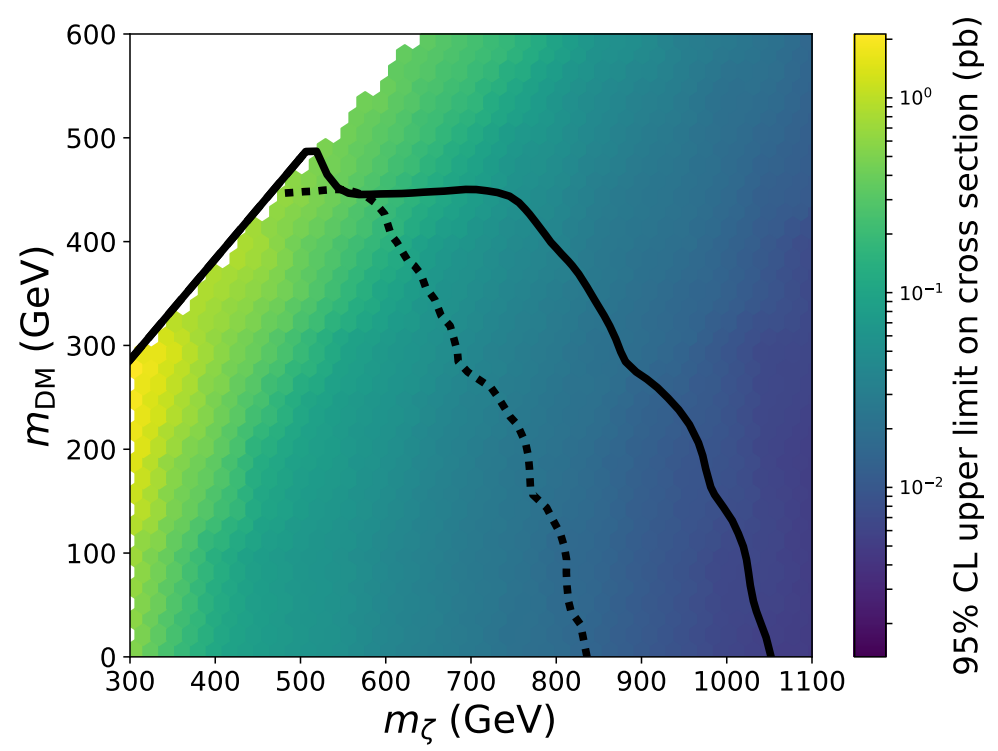

Figure 2: Exclusion limit at 95\% CL on the cross section for $\zeta$ pair production from the CMS data [28] based on $35.9 \mathrm{fb}^{-1}$ at $13 \mathrm{TeV}$. The region below the solid line corresponds to the excluded region for the case of fermion DM ( $N_{0}$ is the DM particle) whereas the region below the dashed line is the excluded region for the case of scalar DM (when $\chi_{0}$ is the DM particle).

the current excluded region in the $\left(m_{N_{0}}, m_{\zeta}\right)$ plane. At $13 \mathrm{TeV}$ with $36.1 \mathrm{fb}^{-1}$ the ATLAS results [29] are reported taking into account the production of 8 squark states of the first and second generation. Since the results are similar to the ones from CMS [28], we expect similar lower bounds on $m_{\zeta}$.

In the case of scalar dark matter ${ }^{2}, E^{0}$ subsequently decays to $\nu+\chi_{0}$ and $E^{+}$to $\ell^{+}+\chi_{0}$. This leads to the following collider signatures: $2 j+E_{\mathrm{T}}^{\text {miss }}, 2 j+\ell^{ \pm}+E_{\mathrm{T}}^{\mathrm{miss}}$ and $2 j+\ell_{1}^{+} \ell_{2}^{-}+E_{\mathrm{T}}^{\text {miss }}$, with a branching fraction of $25 \%, 50 \%$ and $25 \%$, respectively. These kinds of signals were studied in Run-I by ATLAS at $8 \mathrm{TeV}$ with luminosities of around $20 \mathrm{fb}^{-1}$ in the context of simplified supersymmetric models for squark production, assuming $100 \%$ branching frac-

\footnotetext{
${ }^{2}$ We assume $m_{N} \gg m_{E}$.
} 
tions within each signal. The three analysis are orthogonal and set independent limits on the squark and neutralino masses. We are not aware of published searches for first two-generation squarks at Run-II in signals with hard-leptons, jets and missing transverse momentum. ATLAS has searched for new phenomena in events of squark pair production having final states with same-flavor opposite-sign dilepton pair, jets and large missing transverse momentum [30, 31, 32]. The sequence of the decay processes is $\tilde{q} \tilde{q}^{*} \rightarrow\left(q \tilde{\chi}_{2}^{0}\right)\left(q \tilde{\chi}_{2}^{0}\right)$ with $\tilde{\chi}_{2}^{0} \rightarrow \tilde{\ell}^{\mp} \ell^{ \pm} / \tilde{\nu} \nu$ and $\tilde{\ell}^{\mp} / \tilde{\nu} \rightarrow \ell^{\mp} \tilde{\chi}_{1}^{0} / \nu \tilde{\chi}_{1}^{0}$.

By comparing the results from ATLAS for $2 j+E_{\mathrm{T}}^{\mathrm{miss}}$ at $8 \mathrm{TeV}$ [33] which uses a lepton veto, with the results of [31] which uses additional leptons, we can check that the search without leptons has a greater sensitivity. For example, for a neutralino mass of $100 \mathrm{GeV}$, the excluded mass of a eight-fold degenerate squark was $900 \mathrm{GeV}$ without leptons, $860 \mathrm{GeV}$ with opposite sign dileptons, and $800 \mathrm{GeV}$ with one-lepton. In this way, the larger exclusion for the production of squarks is in the signal without further leptons. In the case of scalar dark matter, the branching for two jets and zero leptons is $25 \%$, therefore the bounds discussed above at $13 \mathrm{TeV}$ for this signal become weaker. In particular, we have found that the exclusion for $m_{\zeta}$ goes up to $\sim 800(600) \mathrm{GeV}$ for a DM mass of 100 (400) GeV. The full recast is presented in the lower exclusion curve (dashed line) of Fig. 2 .

In our model the greater exclusion happens when the mass of $E^{+}$is close to the $\zeta$ mass, such that the jets are sufficiently soft, so that the signal becomes effectively $\ell^{+} \ell^{-}+E_{\mathrm{T}}^{\text {miss }}$ without jets. In such a case, we can recast the searches for simplified supersymmetric models with slepton pair production. The results of searches for the first two generations of sleptons at Run-II in signals with opposite sign dileptons and missing transverse momentum for $36.1 \mathrm{fb}^{-1}$ are reported by ATLAS in [34]. Taking into account the $25 \%$ branching into the charged lepton and the scalar dark matter particle, the exclusion region at $13 \mathrm{TeV}$ covers mass values up to $m_{\zeta} \sim 1400 \mathrm{GeV}$ for $m_{\chi_{0}} \lesssim 800 \mathrm{GeV}$. In Fig. 3, we present the full recasted 


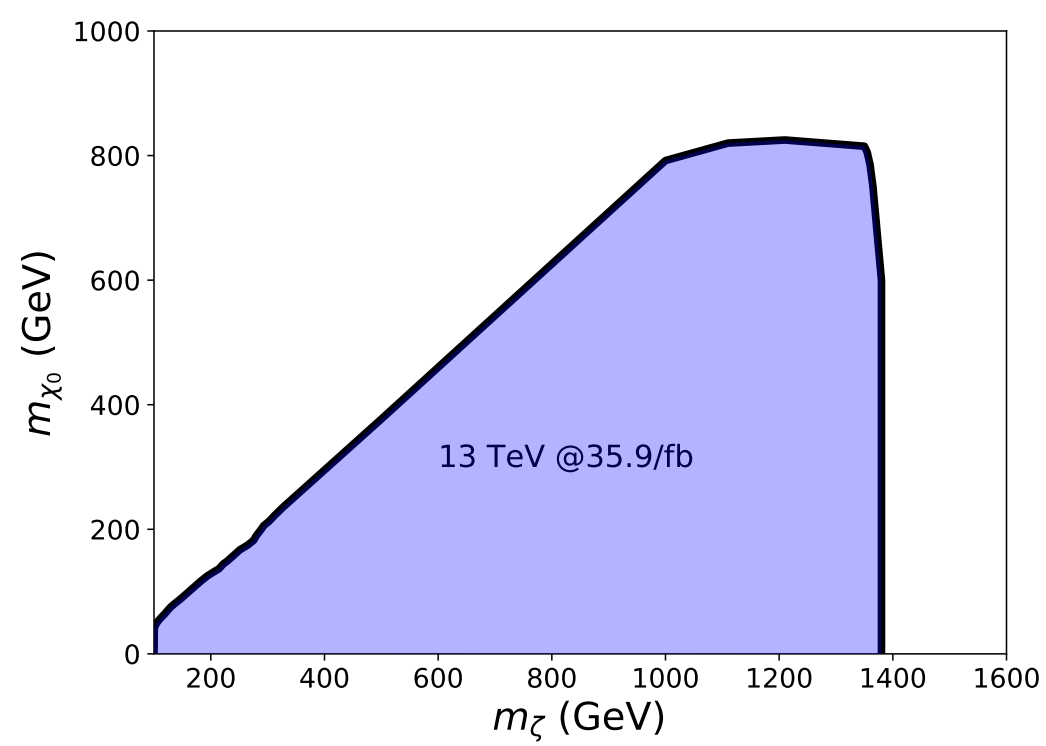

Figure 3: Excluded region (below the solid line) for a singlet scalar quark decaying into a signal which becomes effectively of opposite-sign dileptons and missing transverse momentun. This is obtained from a recast of preliminary results of data for search of dileptons and missing transverse momentun at $13 \mathrm{TeV}$ by ATLAS [34] by using a luminosity of $35.9 \mathrm{fb}^{-1}$.

exclusion region at $13 \mathrm{TeV}$.

The limits with one additional lepton studied in [31] are applicable to the case where $N_{L}$ is the lightest or the next to lightest neutral fermion, since this would correspond to an intermediate $\tilde{\chi}_{2}^{0}$ which decays into $\chi_{1}^{0}$ with further gauge bosons. Therefore, we would expect softer bounds in this case.

Conclusion: A new insight as to the nature of lepton number has been proposed. It is identified with the Peccei-Quinn symmetry which solves the strong CP problem, with the appearance of an invisible axion. A residual symmetry remains, i.e. $(-1)^{L}$, which serves also as dark parity, i.e. $(-1)^{3 B+L+2 j}$. New particles which are odd under this $Z_{2}$ allow the one-loop radiative generation of neutrino masses, and provide a weak-scale component of 
dark matter in addition to the axion. We show how the two sectors may be connected with a new singlet scalar quark $\zeta$, which may be easily probed (or discovered in the future) at the LHC through its subsequent decays to either the fermion or scalar dark-matter candidate.

Acknowledgements : The work of E. M. has been supported in part by the U. S. Department of Energy under Grant No. de-sc0008541. The work of D. R. and O. Z. has been partly supported by the Grants Sostenibilidad-GFIF and CODI-IN650CE, and by COLCIENCIAS through the Grant No. 111-565-84269.

\section{References}

[1] See for example E. Ma, Phys. Rev. Lett. 81, 1171 (1998).

[2] E. Ma, Phys. Rev. D73, 077301 (2006).

[3] E. Ma, Phys. Rev. Lett. 115, 011801 (2015).

[4] B. Dasgupta, E. Ma, and K. Tsumura, Phys. Rev. D89, 041702(R) (2014).

[5] R. Peccei and H. R. Quinn, Phys. Rev. Lett. 38, 1440 (1977).

[6] For a review, see for example J. E. Kim and G. Carosi, Rev. Mod. Phys. 82, 557 (2010).

[7] S. Weinberg, Phys. Rev. Lett. 40, 223 (1978).

[8] F. Wilczek, Phys. Rev. Lett. 40, 279 (1978).

[9] S. Fraser, E. Ma, and O. Popov, Phys. Lett. B737, 280 (2014).

[10] D. Restrepo, A. Rivera, M. Sanchez-Pelaez, O. Zapata, and W. Tangarife, Phys. Rev. D92, 013005 (2015).

[11] E. Ma, Phys. Rev. Lett. 112, 091801 (2014). 
[12] E. Ma, Phys. Lett. B741, 202 (2015).

[13] J. E. Kim, Phys. Rev. Lett. 43, 103 (1979).

[14] M. A. Shifman, A. Vainshtein, and V. I. Zakharov, Nucl. Phys. B166, 493 (1980).

[15] P. Sikivie, Phys. Rev. Lett. 48, 1156 (1982).

[16] G. Raffelt and D. Seckel, Phys. Rev. Lett. 60, 1793 (1988).

[17] A. Alves, D. A. Camargo, A. G. Dias, R. Longas, C. C. Nishi, and F. S. Queiroz, JHEP 1610, 015 (2016).

[18] M. Shin, Phys. Rev. Lett. 59, 2515 (1987).

[19] E. Ma, Phys. Lett. B514, 330 (2001).

[20] Q.-H. Cao, S. Khalil, E. Ma, and H. Okada, Phys. Rev. Lett. 106, 131801 (2011).

[21] V. Silveira and A. Zee, Phys. Lett. 161B, 136 (1985).

[22] J. McDonald, Phys. Rev. D 50, 3637 (1994).

[23] C. P. Burgess, M. Pospelov and T. ter Veldhuis, Nucl. Phys. B 619, 709 (2001)

[24] N. Arkani-Hamed, S. Dimopoulos and S. Kachru, hep-th/0501082.

[25] R. Mahbubani and L. Senatore, Phys. Rev. D 73, 043510 (2006).

[26] F. D’Eramo, Phys. Rev. D 76, 083522 (2007).

[27] R. Enberg, P. J. Fox, L. J. Hall, A. Y. Papaioannou and M. Papucci, JHEP 0711, 014 (2007).

[28] CMS Collaboration [CMS Collaboration], CMS-PAS-SUS-16-036. 
[29] The ATLAS collaboration [ATLAS Collaboration], ATLAS-CONF-2017-022.

[30] G. Aad et al. [ATLAS Collaboration], Eur. Phys. J. C 75 (2015) no.7, 318. Erratum: Eur. Phys. J. C 75 (2015) no.10, 463.

[31] G. Aad et al. [ATLAS Collaboration], JHEP 1504, 116 (2015).

[32] G. Aad et al. [ATLAS Collaboration], JHEP 1510, 054 (2015).

[33] G. Aad et al. [ATLAS Collaboration], JHEP 1409, 176 (2014).

[34] The ATLAS collaboration, ATLAS-CONF-2017-039. 